Check for updates

Cite this: RSC Adv., 2017, 7, 44104

Received 10th July 2017

Accepted 4th September 2017

DOI: $10.1039 / c 7 r a 07589 h$

rsc.li/rsc-advances

\section{Optimized PAMAM coated magnetic nanoparticles for simultaneous hyperthermic treatment and contrast enhanced MRI diagnosis $\dagger$}

\author{
A. Boni, (D) *a A. M. Basini, ${ }^{\text {b }}$ L. Capolupo, ${ }^{\text {a }}$ C. Innocenti, ${ }^{c}$ M. Corti, ${ }^{b}$ M. Cobianchi, ${ }^{d}$ \\ F. Orsini, ${ }^{b}$ A. Guerrini, ${ }^{c}$ C. Sangregorio ${ }^{c e}$ and A. Lascialfarib
}

We report the synthesis and characterization of multi-functional monodisperse superparamagnetic Magnetic NanoParticles, MNPs, able to act as contrast agents for magnetic resonance and Magnetic Fluid Hyperthermia (MFH) mediators. The investigated samples are constituted of a magnetic core of magnetite and a biocompatible PAMAM coating. We studied two samples with the same magnetic volume but different shape, i.e. spherical and faceted. Despite the relatively large size (MNPs of $20 \mathrm{~nm}$ diameter) that generally leads to particles' aggregation and instability, the resulting samples were very stable. For faceted MNPs, the efficiency in contrasting Magnetic Resonance images, i.e. the nuclear transverse ${ }^{1} \mathrm{H}$ NMR relaxivity $r_{2}$, reached values of about $250 / 300 \mathrm{mM}^{-1} \mathrm{~s}^{-1}$ at clinical frequencies $f>5$ $\mathrm{MHz}$, i.e. 2.5/3 times higher than the commercial compound Endorem, while the Specific Absorption Rate at $H_{\mathrm{ac}} \sim 10 \mathrm{kA} \mathrm{m} \mathrm{m}^{-1}$ and frequency $f<300 \mathrm{kHz}$ (i.e. within the physiological limits) reaches $900 \mathrm{~W} \mathrm{~g}^{-1}$, suggesting this system as a potentially useful mediator for $\mathrm{MFH}$. The experimental data strongly indicate the new synthesized MNP systems as good candidates for theranostic applications.

\section{Introduction}

Local control of tumors is a major challenge because of the cells' rapid proliferation and the possible diffusion of tumor cells in the whole body with consequent metastases. In the current local treatments of tumors by means of radiotherapy (RT) and chemotherapy (ChT), and related refined techniques (like cyberknife and gamma-knife), the side-effects are severe. For many tumor sites, ${ }^{1-3}$ the effectiveness of RT and ChT is significantly enhanced when combined with hyperthermia (HT), i.e. raising tissue temperature up to $43-45{ }^{\circ} \mathrm{C}$. Indeed, HT has been shown to improve the clinical outcome when added to $\mathrm{RT}^{\mathrm{T}}$ and $\mathrm{ChT}^{4-6}$ or alone. $^{7}$ In addition to a reduction in tumor bulk and analgesia requirements, the thermal treatment as coadjuvant treatment improves quality of life with decreased pain and preserves patient's function and appearance. Further advantages include a reduction in procedural costs, avoidance of complex repetitive

${ }^{a}$ Center for Nanotechnology Innovation @NEST, Istituto Italiano di Tecnologia, Piazza San Silvestro, 12 - 56127 Pisa, Italy.E-mail: adriano.boni1@virgilio.it ${ }^{b}$ Dipartimento di Fisica and INSTM, Università degli studi di Milano, Italy

'Department of Chemistry "Ugo Shiff", University of Florence and INSTM, via della Lastruccia 3 - 50019 Sesto F. no, Firenze, Italy

${ }^{d}$ Dipartimento di Fisica and INSTM, Università degli studi di Pavia, Italy ${ }^{e}$ CNR-ICCOM and INSTM, via Madonna del Piano, 50019 Sesto F. no, Firenze, Italy $\dagger$ Electronic supplementary information (ESI) available. See DOI: $10.1039 / \mathrm{c} 7 \mathrm{ra07589h}$ surgeries, and the ability to visualize the area during the treatment.

Among various methods of hyperthermia, Magnetic Fluid Hyperthermia (MFH) has drawn considerable attention. ${ }^{8-10}$ In MFH, fluids containing magnetic nanoparticles (MNPs) (generally iron oxide based systems) are delivered to the cancer and then heated by on external alternating magnetic field, resulting in hyperthermic treatment of cancer tissue. Magnetic nanoparticles based therapy has been investigated for many types of tumors, including those of the central nervous system. ${ }^{11,12}$ Clinical trials (Phase I and II) on glioblastoma multiforme and prostate cancers have already been performed ${ }^{\mathbf{1 3}}$ and, at present, Phase III clinical study on the first is going on. Concerning glioblastoma, the survival time of patients has been increased by a factor 1.5/2 after MFH application. This result stimulated a lot of multidisciplinary research all over the world involving physicists, chemists, engineers, biologists and physicians, often starting from basic proprieties of novel nanoparticles to experimental tests on cells cultures and animal models.

MNPs have also been proposed as complimentary or alternative Magnetic Resonance Imaging (MRI) contrast agents with respect to the paramagnetic Gadolinium-Based Contrast Agents (GBCAs), thanks to their ability to increase the transverse relaxation rate $\left(r_{2}\right)$ and bring negative contrast. ${ }^{14}$ The general idea about MNPs is to obtain a nanosystem that can work as therapeutic $\mathrm{MFH}$ agent due to its heat release capacity, and as diagnostic agent because of the contrast efficiency in the MR 
images. Other functionalities, like fluorochromes for optical imaging or carrier for drug loading, can be additionally envisaged.

On this purpose, some of us recently developed a new class of iron oxide nanoparticles coated with lipid modified Poly (AMidoAMine) (PAMAM) dendrimers. ${ }^{15,16}$ This approach for the coating of MNPs, which occurs through hydrophobic interaction of the $\mathrm{C}_{12}$ chains of the dendrimer with the oleic acid coating, presents important advantages over existing strategies. Firstly, in many case ${ }^{17}$ the coating may interact with the surface atoms of the magnetic core to form a nonmagnetic layer, reducing its effective size and the total amount of magnetic phase. The PAMAM based procedure does not require harsh conditions, thus preserving magnetic and structural properties of the iron oxide nanoparticles, which can be finely tuned by synthesizing the nanoparticles with the decomposition of organometallic precursors. Moreover, the possibility of performing the coating process directly in water/pentane makes possible to avoid toxic solvents such as dimethyl sulfoxide and $\mathrm{N}, \mathrm{N}$-dimethyl formamide, which are difficult to remove, thus reducing the safety risks deriving from their presence in traces in the final injectable solution. Finally, the mild temperatures required by this method make possible to use dendrimers modified with thermally unstable moieties through the surface amino residues, thus extending the range of functional groups that can be conjugated to MNPs for targeting or sensing. In vitro cell viability tests, performed by administration to bEND 5 cells and measurement of metabolic activity, the poor toxicity of this material was proved at least up to $1000 \mathrm{ng} \mathrm{mL}^{-1}$ of iron, obtaining results similar to those of widely used coatings, such as 3-aminopropyltriethoxysilane (APTES) over a wide size range (4-14 nm). ${ }^{18}$ Further on, Dynamic Light Scattering (DLS) measurements of hydrodynamic size and relaxometric mesurements repeated on the same sample over weeks provided evidence of a high colloidal stability both in water and in biological media, such as human plasma. Additionally, these MNPs demonstrated to induce a strong reduction in the MR signals in the rat liver after injection, suggesting their potential use as $T_{2}$ MRI contrast agents with enhanced sensitivity.

In this work we aim to investigate the possibility to tune the properties of PAMAM coated MNPs to obtain a material which can simultaneously provide hyperthermic efficacy and increase the contrast in MRI imaging. In order to achieve this goal and maximize both the hyperthermic and relaxometric efficacy, we decided to synthesize samples, having the highest diameter obtainable with this method ( $c a .20 \mathrm{~nm}$ ), of two different shapes (spheres and faceted-like) and we performed a full structural, magnetic and relaxometric characterization. As a final test, we measured the Specific Absorption Rate (SAR).

\section{Experimental}

\subsection{Sample preparation}

In the preparations, iron pentacarbonyl $\left(\mathrm{Fe}(\mathrm{CO})_{5}, 99.5 \%\right)$, oleic acid (OA), dioctyl ether, trimethylamine $N$-oxide, toluene, ethanol, anhydrous dimethylsulfoxide (DMSO), anhydrous $\mathrm{N}, \mathrm{N}$ dimethylformamide (DMF), PAMAM- $\mathrm{C}_{12}$ generation 4 (methanol solution) were purchased from Sigma-Aldrich. Oleic acid coated samples were prepared according to the reported method: $0.2 \mathrm{~mL}$ of iron pentacarbonyl were decomposed in $20 \mathrm{~mL}$ of dioctyl ether in the presence of oleic acid (1:3 Fe/oleic acid molar ratio). Further addition of iron oleate, prepared by decomposition of $0.2 \mathrm{~mL}$ of iron pentacarbonyl at $130^{\circ} \mathrm{C}$ in the presence of oleic acid (1:3 Fe/oleic acid molar ratio), in dioctylether at solvent reflux temperature for 3 hours allowed growing the particle size. Oxidation with trimethyl amine $N$ oxide was performed before and after the growth step (A1) or only at the end of the growth (A2).

The coating modification was performed according to the reported method: ${ }^{15}$ NP-OAs (13 mg, $\left.83 \mu \mathrm{mol} \mathrm{Fe,} 30 \mu \mathrm{mol} \mathrm{OA}\right)$ were added to a solution of $0.25 \mathrm{~mL}$ of PAMAM- $\mathrm{C}_{12}$ generation 4 (methanol solution, $20 \mathrm{mg}, 1 \mu \mathrm{mol}$ ) in $10 \mathrm{~mL}$ of water. After 16 hours stirring, solvents were removed under vacuum and replaced by water. 30 minutes of sonication facilitated the dispersion of the powder in water solution, which became orange. Recovery with a magnet allowed removing the unreacted dendrimers and other residues of the reaction.

\subsection{Structural and magnetic characterization}

The measurement of iron content in the samples was performed by an Inductively Coupled Plasma-Mass Spectrometry technique (ICP-MS, Agilent Technologies Inc., 7700 series, USA). DLS measurements were performed on a Beckman \& Coulter Delsa Max instrument at $298 \mathrm{~K}$. Conventional TEM micrographs and electron diffraction patterns were obtained with a Zeiss Libra 120 TEM operating at $120 \mathrm{kV}$ and equipped with in column Omega filter for Electron-Energy Loss Spectroscopy (EELS). The MNP size distribution histogram was determined using enlarged TEM micrographs taken at magnification of $100 \mathrm{~K}$ on a statistical sample of ca. 200 MNPs. Magnetization data were collected on dry powder with a Quantum Design Ltd. SQUID magnetometer working in the temperature range of $1.8-350 \mathrm{~K}$ and the magnetic field range of 0-5 T.

\subsection{Relaxometry}

Water ${ }^{1} \mathrm{H}$ NMR relaxation measurements at fixed frequency $10<$ $\nu<60 \mathrm{MHz}$ were collected by means of a Stelar SpinMaster Spectrometer (Stelar S.n.c., Mede (PV), Italy) on water solution of PAMAM-magnetic particles. Water proton $T_{1}$ data were obtained by means of the Inversion-Recovery technique (16 experiments, 2 scans). Water proton $T_{2}$ data were obtained by means of the CPMG sequence with the following parameters: 8 scans, 2048 data points, delay time equal to 5 times $T_{1}$, applying a phase cycle on the pulses. Data were fitted with a monoexponential function.

${ }^{1}$ H-NMRD (Nuclear Magnetic Relaxation Dispersion) profiles for $10 \mathrm{kHz}<\nu<10 \mathrm{MHz}$ (i.e. magnetic field strengths in the range $2.4 \times 10^{-4}$ to $0.23 \mathrm{~T}$ ) were recorded at $37^{\circ} \mathrm{C}$ on a StelarSpinmaster-FFC field-cycling relaxometer (StelarS.n.c., Mede (PV), Italy) by measuring the water proton longitudinal and transverse relaxation rates with the use of FFC proper sequences. 
Phantoms consisting of PAMAM-USPIO solution in vials were used to acquire Magnetic Resonance images through an ESAOTE Artoscan system operating at a fixed magnetic field of 0.2 Tesla, i.e. $\sim 8.5 \mathrm{MHz}$. The longitudinal $\left(T_{1}\right)$ relaxation time of the solutions was measured using a $T_{1}$-weighted MRI sequence based on a standard Inversion Recovery (IR) with the following parameters: $T_{\mathrm{R}}=160 \mathrm{~ms}, T_{\mathrm{E}}=26 \mathrm{~ms}$, slice thickness $=3 \mathrm{~mm}$. The used $T_{2}$-weighted sequence was a spin-echo with experimental parameters: $T_{\mathrm{E}}=80 \mathrm{~ms}, T_{\mathrm{R}}$ (Repetition Time) $=5000 \mathrm{~ms}$ and $T_{\mathrm{H}}$ (slice thickness) $=3 \mathrm{~mm}$.

\subsection{Calorimetric measurements}

The hyperthermic efficiency was evaluated with a Magnetherm system by nanoTherics Ldt., which allows investigating frequencies in the range $100-1000 \mathrm{kHz}$ with applied alternating magnetic fields of intensity 4-20 mT. An optical fiber by Optocon AG was used to measure the temperature with $1 \mathrm{~s}$ recording time. For all the experiments, the initial temperature was approximately $21 / 22{ }^{\circ} \mathrm{C}$ (laboratory conditions).

The initial slopes of the temperature kinetics curves, $T(t)$, were obtained by using the Box-Lucas fit reported in eqn (1):

$$
T_{\mathrm{f}}=T_{\mathrm{i}}+\frac{B}{A}\left(\mathrm{e}^{A t}-1\right)
$$

where $T_{\mathrm{i}}$ and $T_{\mathrm{f}}$ are the initial and final temperatures and parameters $A$ and $B$ are related to the heat exchange with the surrounding environment and the heating generated by the nanoparticles in the solution, respectively. ${ }^{19}$

Before the measurements, the samples were sonicated for 5 minutes and then thermalized to the laboratory temperature before starting the experiment.

\section{Results and discussion}

\subsection{Synthesis and structural characterization}

Iron oxide nanoparticles were synthesized according to the method proposed by Hyeon and coworkers. ${ }^{20}$ The obtained nanoparticles were grown by further reaction with iron oleate. In the case of sample A1, the nanoparticles were oxidized twice, before and after the growth step by addition of trimethyl amine $N$-oxide, while in the case of sample A2 the nanoparticles were oxidized only after the growth step.

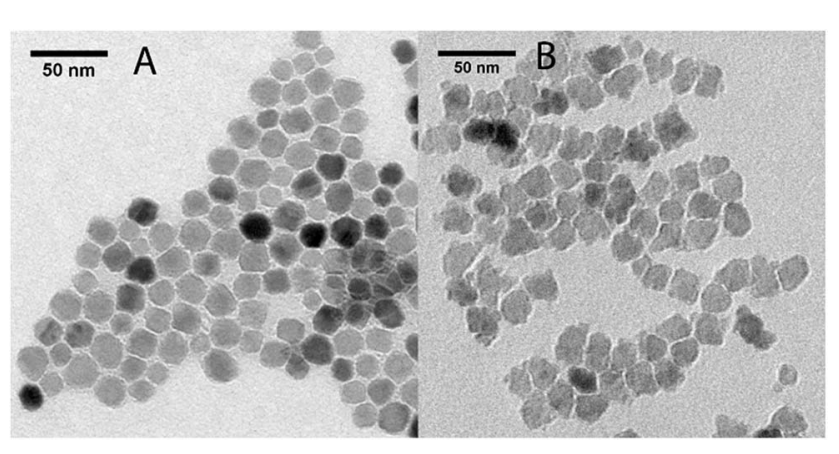

Fig. 1 TEM images of samples A1 (left) and A2 (right).
The final products were characterized by TEM analysis (Fig. 1). A1 consisted of a dispersion of regular-shaped particles, approximated by spheres, while A2 showed an irregular and faceted shape. The size distribution over $c a$. 200 nanoparticles for each sample have been fitted to a Gaussian to obtain the mean diameters, which are reported in Table 1 (distribution graphs are reported in ESI, Fig. S1 $\dagger$ ).

The coating procedure was performed on the samples $\mathrm{A}(1-2)$ by means of the reaction with lipid-modified PAMAM dendrimers of generation 4, which allowed us to obtain stable water dispersions for both samples, which were re-named $\mathrm{S}(1-2)$, respectively. The final water dispersions were characterized by DLS, TEM and ICP-MS. From the DLS mass distributions (see ESI, Fig. S2†), it was observed that the average hydrodynamic diameter was about $10 \mathrm{~nm}$ higher than the pristine magnetic core A(1-2) size (cfr $d_{\text {core }}$ and $d_{\text {hyd }}$ in Table 1 ). By considering that the molecular diameter of the used generation 4 PAMAM on the surface is $4.5 \mathrm{~nm},{ }^{21}$ this result is consistent with TEM analysis on S(1-2), which did not show evidences of consistent aggregates formation (see ESI, Fig. S3†). Further DLS measurements repeated over weeks did not report changes in mean hydrodynamic diameter and confirmed the dispersions stability. In conclusion, the morphological and structural analysis on S(1-2) allowed verifying that the coating step did not alter the main properties of the samples, as already demonstrated in our previous work. ${ }^{15}$

\subsection{Magnetic characterization}

In order to establish the magnetic behavior of the MNP dispersions, which strongly influence their relaxometric performance, a full set of magnetic measurements was performed on the solid (dry powders) samples A1-A2. The main magnetic parameters obtained from the measurements are summarized in Table 1. The zero-field cooled/field cooled (ZFC/ FC) magnetization curves, collected with a probe field of $5 \mathrm{mT}$ in the 5-300 $\mathrm{K}$ temperature range, are reported in Fig. 2A.

The curves represent the typical behavior of a set of single domain MNPs. Sample A1 also displays a smoothed peak at ca. $110 \mathrm{~K}$, which can be associated to the Verwey transition, whose characteristic temperature for bulk magnetite $(120 \mathrm{~K})$, is often reduced in nanosized systems. This transition is typical of magnetite and its absence in the ZFC/FC curve of A2 can be ascribed to a certain degree of oxidation to maghemite. The average blocking temperature, $T_{\mathrm{B}}$, commonly associated to the temperature at which the ZFC curve reaches its maximum, is about $270 \mathrm{~K}$ for A2, while it could not be estimated accurately for $\mathrm{A} 1$ as it is above the temperature range of the instrument. According to the Néel model for non-interacting MNPs, $T_{\mathrm{B}}$ is proportional to the anisotropy energy barrier $\Delta E=K V$, where $K$ is the anisotropy constant of the material and $V$ the mean magnetic volume of the NPs. Thus, the observed difference may be ascribed to a difference in anisotropy and/or magnetic volume between the two samples.

The magnetization as a function of the applied field, $M(H)$, was measured at $300 \mathrm{~K}$ and $2.5 \mathrm{~K}$ (Fig. 2C) up to $5 \mathrm{~T}$. The magnetization curves rapidly approach the saturation. 
Table 1 Structural parameters: core (by TEM) and hydrodynamic mean diameters (by DLS, measured on the respective water suspensions S1 and S2). Magnetic parameters of the dry powders extracted from ZFC/FC and $M(H)$ data: blocking temperature $T_{\mathrm{B}}$, coercive field $H_{\mathrm{C}}$, reduced remanent magnetization $M_{R}$ and saturation magnetization $M_{S}$ at low and high temperature. $M_{S}$ values are reported per gram of magnetic material, $\mathrm{Fe}_{3} \mathrm{O}_{4}$, as determined by the ICP-MS analysis

\begin{tabular}{llllllll}
\hline Sample & $d_{\text {core }}(\mathrm{nm})$ & $d_{\text {hyd }}(\mathrm{nm})$ & $T_{\mathrm{B}}(\mathrm{K})$ & $\mu_{0} H_{\mathrm{C}}(\mathrm{Oe})$ & $M_{\mathrm{R}} / M_{\mathrm{S}}$ & $\begin{array}{l}\mathrm{MS}\left(\mathrm{emu} \mathrm{g}^{-1}\right) \\
\mathrm{K}\end{array}$ & $\begin{array}{l}\mathrm{MS} @ 300 \mathrm{~K} \\
\left(\mathrm{emu} \mathrm{g}^{-1}\right)\end{array}$ \\
\hline A1(S1) & $19.5 \pm 1.8$ & $30.2 \pm 3.3$ & 270 & 324 & 0.28 & 77.2 & 69.8 \\
A2(S2) & $19.7 \pm 0.8$ & $33.7 \pm 3.8$ & $>300$ & 442 & 0.33 & 91.2 & 78.8 \\
\hline
\end{tabular}
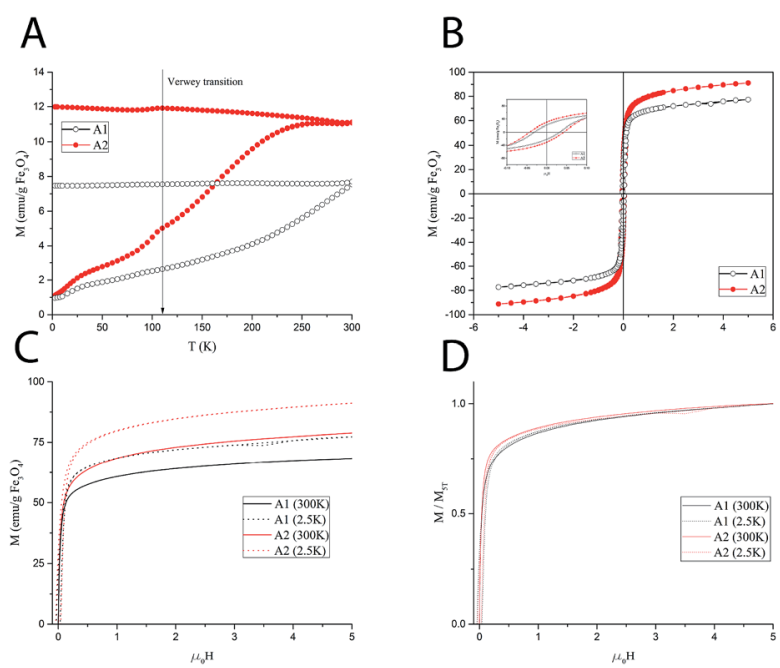

Fig. 2 (A) ZFC and FC magnetization curves acquired by applying a $5 \mathrm{mT}$ probing field; (B) hysteresis cycles at $T=2.5 \mathrm{~K}$; (C) $M(H)$ curves measured at 2.5 and $300 \mathrm{~K} ;(\mathrm{D}) \mathrm{M}(\mathrm{H})$ curves measured at 2.5 , and $300 \mathrm{~K}$ normalized to the $5 \mathrm{~T}$ value. All the data were collected on dry powders of samples $\mathrm{A} 1$ and $\mathrm{A} 2$ and are reported per gram of magnetic material, $\mathrm{Fe}_{3} \mathrm{O}_{4}$, evaluated from ICP-MS data.

However, as the magnetization curves do not completely saturate, an estimate of the saturation value, $M_{\mathrm{S}}$, was obtained by fitting the high field magnetization data to the empirical law $M=M_{\mathrm{S}}+b / H+c / H^{2} .{ }^{22}$ At high temperature, the saturation values are 69.8 and $78.8 \mathrm{emu} \mathrm{g}^{-1}$ of iron oxide for A1 and A2, respectively.

Hysteresis was observed at low temperature (Fig. 2B), while no coercivity is present at $300 \mathrm{~K}$, as expected for superparamagnetic nanoparticles. The remanent magnetization, $M_{\mathrm{R}}$, and the coercive field, $H_{\mathrm{C}}$, are slightly higher for sample A2. The magnetic anisotropy constant, $K$, can be estimated from the low temperature coercive field $H_{\mathrm{C}}$ as, for an ensemble of randomly oriented NPs at a finite temperature, is given by $\mu_{0} H_{\mathrm{C}}=$ $0.96\left(K / M_{\mathrm{S}}\right)\left[1-\left(T / T_{\mathrm{B}}\right)^{0.77}\right]$. In our case, since the temperaturedependent term is negligible for all samples $\left(T_{\mathrm{B}}\right.$ is much larger than $T=2.5 \mathrm{~K}$ ), the anisotropy constant can be simply estimated by $K=H_{\mathrm{C}} M_{\mathrm{S}} / 0.96$ where $M_{\mathrm{S}}$ is the value at $2.5 \mathrm{~K}$. The results reported in Table 1 show a higher anisotropy for A2. Hence, in principle, the higher $T_{\mathrm{B}}$ of A1 should be associated with a higher average magnetic volume. Furthermore, in the normalized magnetization curves (Fig. 2D), a faster approach to saturation can be observed for sample A1. This suggests a higher susceptibility, which is commonly associated to a higher magnetic volume. Nevertheless, the mean volume foreseen by the Nèel simple model to compensate the anisotropy difference should be too large to be realistic. A more complex model including other factors (size distribution and interparticle interactions) should be envisaged to account for $T_{\mathrm{B}}$ findings, which is beyond the purpose of the present work.

The magnetic measurements were repeated on a lyophilized sample of S2 to test the effect of the PAMAM coating on the magnetic behavior. The comparison between A2 and S2 (see ESI, Fig. S4 $\dagger$ ) shows small differences, which can be ascribed to the change of the interparticle interactions rather than to an intrinsic modification of the particle surface during the coating procedure. In particular, the decreased dipolar interactions due to the larger interparticle distance provided by the coating layer can account for the small reduction of the blocking temperature of S2 (253 K).

The superparamagnetic behavior at room temperature of both samples, particularly evidenced for sample A2, together with the high $M_{\mathrm{S}}$ values of the material, prefigures the use of these nanoparticles as negative contrast agents for MRI, as this allows a fast response to the applied magnetic field and a large and constant magnetic moment for single particles. ${ }^{23}$

\section{3 $\quad{ }^{1} \mathrm{H}$ NMR relaxometric characterization}

A relationship between the dynamic and static magnetic properties of the samples has been evaluated through the relaxometric behavior of the ${ }^{1} \mathrm{H}$ nuclei present in the nanoparticle solution (i.e. water's hydrogens). The nuclear magnetic relaxation properties of a compound are ideally obtained by the study of its Nuclear Magnetic Resonance Dispersion (NMRD) profile. These curves give the relaxivity evolution of the nuclear spins of the ${ }^{1} \mathrm{H}$ versus the external magnetic field, and constitute an interesting tool to optimize the parameters of the nanoparticle synthesis ${ }^{24}$ for having better MRI contrast agents. The relaxivity $r_{i}$ $(i=1,2)$ is defined as the increase of the relaxation rate $\left(r_{i}\right.$, the inverse of the relaxation time $T_{i}$ ) of the hydrogen nuclei of $\mathrm{H}$ in the solvent (water in this case) induced by $1 \mathrm{mM}$ of magnetic center (iron in this case). The capability of the nuclear spin of ${ }^{1} \mathrm{H}$ to exchange energy with the giant electronic spin of the nanoparticles increases the nuclear spin relaxivity and gives information about the local electronic spin dynamics. Two different phenomena can be distinguished with respect to the direction of the external static magnetic field. The relaxation of the longitudinal nuclear magnetization is mainly due to the interaction between the lattice and the nuclear spins and $T_{1}$ is the time of equilibrium recovery. The relaxation of the nuclear transverse 
competition $\left(T_{2}\right)$ is the result of the spin-spin interaction. In Magnetic Resonance Imaging (MRI) the signal is contrasted, with different efficacies, by introducing contrast agents that reach specific sites and change locally the relaxivities of the ${ }^{1} \mathrm{H}$ nuclei contained in the human tissues. The $T_{1}$ and $T_{2}$ relaxivities depend on the magnetic field whose typical values in MRI apparatus of hospital are between $0.2 \mathrm{~T}(8.5 \mathrm{MHz})$ and $3 \mathrm{~T}$ $(120 \mathrm{MHz})$. It is worth to notice that in this range of field the MNPs display quite high $r_{1}$ but very high $r_{2}$ so that they can be helpful mainly to increase the $T_{2}$ negative contrast (i.e. local dispersion of signal) in MRI images. For these reasons from now on, we will refer to $r_{2}$ as the contrast efficiency. Fig. 3 shows the longitudinal (A) and transversal (B) relaxometric profiles of samples $\mathrm{S}(1-2)$. All the measured samples present a dispersion curve strongly dominated by the effects of superparamagnetic relaxation, characterized by a low field plateau followed by a smoothed peak in the $0.5-1 \mathrm{MHz}$ range, referred to as the Curie peak, and by a decrease at high fields (i.e. high Larmor frequencies). It can be noticed that sample $\mathrm{S} 1$ presents an irregular $r_{2}$ profile with respect to $\mathrm{S} 2$; the difference is of uncertain origin. The NMRD profiles the two samples present very different MRI contrast efficiency (i.e. $r_{2}$ especially at high fields), in particular S2 displaying higher $r_{2}$ with respect to S1. If it is assumed that the $r_{2}$ value is dominated by the effect of the giant magnetic moment, these data are in agreement with the magnetic measurements, where A2 displayed a higher $M_{\mathrm{S}}$ compared with A1. It is worth to notice that sample S2 presents a transversal relaxivity more than two times higher than the commercial contrast agent Endorem, while the longitudinal relaxivities for $f>10 \mathrm{MHz}$ are comparable. From the relaxometric analysis we can then conclude that the study of local spin dynamics confirms the superparamagnetic regime at room temperature and demonstrates that $\mathrm{S} 2$ is a promising $T_{2}$ contrast agent.

To obtain further information on the microscopic features and the Néel relaxation time of our nanostructures, we fitted the $r_{1}$ and $r_{2}$ profiles by using a heuristic model developed by Roch, Muller and Gillis. ${ }^{25}$ This RMG model considers the nuclear relaxation composed by different contributions: the first due to hyperfine field fluctuations at the nuclear sites caused by the diffusion of solvent protons into the inhomogeneous magnetic

\section{A}

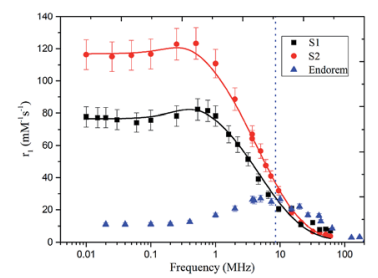

B

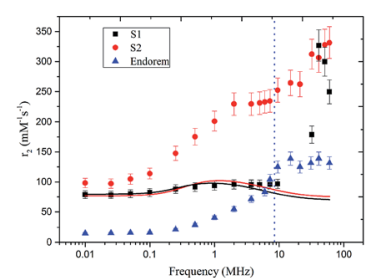

Fig. 3 Nuclear magnetic relaxation dispersion (NMRD) $r_{1}(\mathrm{~A})$ and $r_{2}$ (B) profiles for samples S1, S2 compared to the commercial compound, Endorem. The dashed lines represent the frequencies used in MRI images. The solid lines are the best-fitting to the experimental data (see text for details). field created by the large magnetic moments of the MNP (named Curie relaxation) and the second due to the fluctuations of the magnetic moment of each particle induced by the Néel relaxation with typical correlation time $\tau_{\mathrm{N}}$ (low $f$ ). The model presents a very complicate formula to fit the experimental data (see ref. 25), which is not useful to be reported here. However, the parameters of our interest (reported in Table 2) can be summarized as follows: $\tau_{\mathrm{N}}$ is the Néel relaxation time, $d_{\mathrm{NMR}}$ is the minimum approach distance (i.e. the capacity of water molecules to permeate the coating) and $P$ and $Q$ are weighting factors, bonded by the constraint $P+Q=1$ and related to the magnetic anisotropy (the two limit cases are $P=1$ for null anisotropy energy and $Q=1$ for anisotropy energy tending to infinite). From the fitting of the longitudinal relaxivity $r_{1}$ with RMG model (Fig. 3A, solid lines), we obtained that: (i) the $P / Q$ ratio decreases with increasing anisotropy, coherently with magnetic anisotropy constant data obtained by magnetometric measurements (Table 2); (ii) the values of the Neel relaxation times at room temperature, $\tau_{\mathrm{N}}^{\mathrm{NMR}}$, are in the typical range of SPM compounds reversal time; (iii) the distance of minimum approach $d_{\mathrm{NMR}}$ is intermediate between the magnetic core diameter as determined by TEM and the hydrodynamic diameter $d_{\text {hyd }}$, a result suggesting that our samples' coating is partially penetrated by the water. On the other hand, the experimental $r_{2}$ profile cannot be fitted to the RMG model, the bigger disagreement being in the high field (frequency) region (Fig. 3B, solid lines). A similar mismatch has previously been observed ${ }^{26,27}$ and attributed to one or more physical mechanisms not included in the model itself. On this respect, it should be observed that the RMG model describes systems in the so called motional averaging regime (in short MAR, corresponding to fast motions) while our systems are out of the limit of the MAR regime. In particular they can be considered between the MAR and the SDR (static dephasing regime, slow motions regimes). ${ }^{28}$ In fact, by using the approximated model of Vuong et al. ${ }^{28}$ one can easily guess that our $r_{2}$ experimental data at frequencies between 20 and $60 \mathrm{MHz}$ fall in between the predictions of the different regimes $\left(r_{2}^{\mathrm{MAR}} \sim 72 \mathrm{~s}^{-1} \mathrm{mM}^{-1}, r_{2}^{\mathrm{SDR}} \sim\right.$ 780-800 $\mathrm{s}^{-1} \mathrm{mM}^{-1}$ ). The discussion on further details of the physical mechanisms responsible for the high values of $r_{2}$ in our samples is out of the scope of the present manuscript (Table 3).

\subsection{MR-imaging}

To validate the previous results regarding the high $r_{2}$ contrast efficiency of S1 and S2, we collected MRI images at a field

Table 2 Fitting parameters obtained from the analysis of ${ }^{1} \mathrm{H}-\mathrm{NMRD} r_{1}$ profiles employing Roch's model. The last two columns refer to the distance (multiplied by a factor 2) of minimum approach between the protons of the solution and the MNP centers obtained by NMR, and to the hydrodynamic diameter (reported here again for clarity) extracted from DLS measurements, respectively

\begin{tabular}{lcclll}
\hline & \multicolumn{5}{c}{$r_{2} / r_{1}(60$} \\
Sample & $P / Q$ & $\tau_{\mathrm{N}}^{\mathrm{NMR}} 300 \mathrm{~K}$ & $\mathrm{MHz})$ & $d_{\mathrm{NMR}}(\mathrm{nm})$ & $d_{\mathrm{hyd}}(\mathrm{nm})$ \\
\hline S1 & 2.62 & $1.57 \times 10^{-7}$ & 35.04 & $24.8 \pm 2.0$ & 30.2 \\
S2 & 1.17 & $2.77 \times 10^{-7}$ & 87.23 & $25.1 \pm 0.4$ & 33.7
\end{tabular}


Table 3 Relaxivity values at $8.5 \mathrm{MHz}$ from FFC (first two columns) and MRI (last two columns) techniques. The values are the same within the experimental error

\begin{tabular}{lllll}
\hline Sample & $r_{1}\left(\mathrm{~s}^{-1} \mathrm{mM}^{-1}\right)$ at $8.5 \mathrm{MHz}$ & $r_{2}\left(\mathrm{~s}^{-1} \mathrm{mM}^{-1}\right)$ at $8.5 \mathrm{MHz}$ & $r_{1}\left(\mathrm{~s}^{-1} \mathrm{mM}^{-1}\right)$ at $60 \mathrm{MHz}$ & $r_{2}\left(\mathrm{~s}^{-1} \mathrm{mM}^{-1}\right)$ at $60 \mathrm{MHz}$ \\
\hline $\mathrm{S} 1$ & $96(8)$ & $9.0(0.7)$ & $249(20)$ \\
$\mathrm{S} 2$ & $27(2)$ & $245(19)$ & $3.8(0.3)$ & $331(26)$ \\
Endorem & $37(3)$ & $114(9)$ & $11(1)$ & $106(8)$
\end{tabular}

intensity of $0.2 \mathrm{~T}(\sim 8.5 \mathrm{MHz})$, commonly used by clinical scanners for the investigation of diseases in articulations. As described in the Experimental section, the vials containing different samples at the same Fe concentration were placed in the center of the detection coil. Fig. 4 shows the $T_{1}$ and $T_{2^{-}}$ weighted images of the prepared samples compared to the commercial contrast agent (CA) Endorem and to water. We expect positive bright contrast in the $T_{1}$-weighted image and negative dark contrast in the $T_{2}$-weighted image.

Fig. 4A shows that Endorem is the best $T_{1}$-CA among the analyzed samples, while Fig. $4 \mathrm{~B}$ confirms the best $r_{2}$ performance for $\mathrm{S} 2$, thus being a better $T_{2}$-CA in comparison with Endorem, as $r_{2}^{\mathrm{S}-2}(8.5 \mathrm{MHz}) \sim 254 \mathrm{~s}^{-1} \mathrm{mM}^{-1}>r_{2}^{\text {Endorem }}(8.5 \mathrm{MHz})$ $\sim 106 \mathrm{~s}^{-1} \mathrm{mM}^{-1}>r_{2}^{\mathrm{S}-1}(8.5 \mathrm{MHz}) \sim 95 \mathrm{~s}^{-1} \mathrm{mM}^{-1}$.

\subsection{Hyperthermic efficacy}

The heating mechanism in magnetic materials is related to the capability to adsorb energy from an external alternating magnetic field (AMF) and to dissipate it in the environmental medium. For MNPs superparamagnetic MNPs, as in our case, the energy losses are due to the lag of the magnetization with respect of the field oscillation which can be related to the an effective relaxation time, $\tau_{\text {eff }}$ (see the definition below). The energy loss power per unit volume (Specific Loss Power, SLP) for randomly oriented superparamagnetic nanoparticles, can be thus represented as in eqn (2):

$$
\mathrm{SLP}=\pi \mu_{0} \chi_{0} H_{0}{ }^{2} f \frac{2 \pi f \tau_{\mathrm{eff}}}{1+\left(2 \pi f \tau_{\mathrm{eff}}\right)^{2}} \frac{1}{\rho}
$$

where $\chi_{0}$ is the static magnetic field susceptibility at field $H_{0}, f$ the frequency of the field and $\rho$ the concentration of nanoparticles.

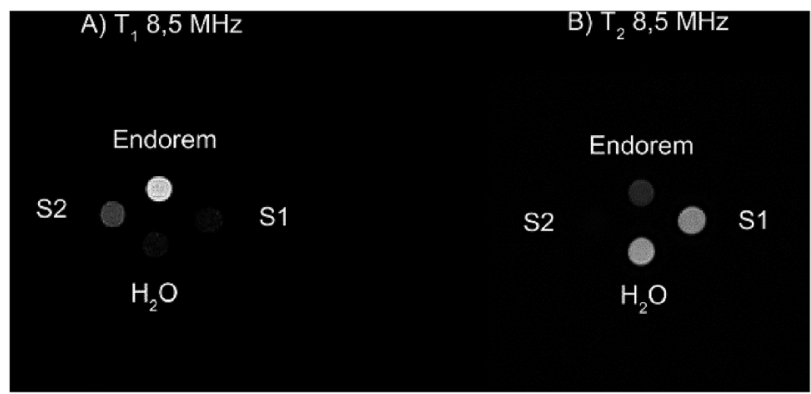

Fig. $4 \mathrm{MRI} T_{1}$-weighted (A) and $T_{2}$-weighted (B) images at $8.5 \mathrm{MHz}$ of (from left and in clockwise) S2, Endorem, S1 and water, this last used as reference (images obtained with the ARTOSCAN by Esaote). All samples (except for water) have the same iron concentration of $0.2 \mathrm{mM}$. The used sequences are reported in the text.
The effective time $\tau_{\text {eff }}$ is given by $\tau_{\text {eff }}=1 / \tau_{N}+1 / \tau_{B}$, where $\tau_{N}$ and $\tau_{\mathrm{B}}$ are the characteristic times of the Néel and Brown mechanisms concurring to the magnetization relaxation, given by:

$$
\tau_{\mathrm{N}}=\tau_{0} \mathrm{e}^{\left(\frac{\Delta E}{k_{\mathrm{B}} T}\right)}
$$

where $\Delta E$ is the anisotropy energy barrier already defined, and $\tau_{0}$ is of the order of $10^{-9} \mathrm{~s}$; and:

$$
\tau_{\mathrm{B}}=3 \eta V_{\mathrm{H}} / K k_{\mathrm{B}} T
$$

where $\eta$ is the viscosity of the carrier fluid, $k_{\mathrm{B}}$ the Boltzmann constant, and $V_{\mathrm{H}}$ the hydrodynamic volume of the particle. As concerns the Nèel mechanism, the magnetization reverses its direction along the MNP's easy axis: when the alternate magnetic field is applied the magnetization is driven far from the equilibrium state and the associated superspin can be thought as rotating and going back to a minimum of energy (along the easy axis). In the Brown mechanism, the whole nanoparticle rotate in the fluid to oriented the nanoparticles easy-axes along the external magnetic field.

The MNPs such as ferrite nanoparticles have shown promising results as potential heating mediators in magnetic hyperthermia due to their efficiency in conversion of magnetic energy into heat even at low concentrations. Magnetic energy dissipation in a ferrofluid sample is measured by calorimetric experiments using the thermodynamic relationship reported in eqn (5):

$$
\mathrm{SLP}=\frac{\Delta T}{\Delta t} \frac{c_{\mathrm{NP}} m_{\mathrm{NP}}+c_{1} m_{1}}{m_{\mathrm{NP}}}
$$

where $m_{\mathrm{NP}}$ and $m_{1}$ are mass of the nanoparticles and the fluid ( $0.11 \mathrm{mg}_{\mathrm{Fe}} \mathrm{mL}^{-1}$ of solution), $c_{1}$ and $c_{\mathrm{NP}}$ is the specific heat of the liquid (water) and the MNP respectively, and $\Delta T / \Delta t$ is the time rate of temperature change.

We measured the temperature kinetics curve $T(t)$ for several of the combinations field-frequency allowed by the experimental set up. We must note that they exceeds the clinical safety limit $\left(f H<4.58 \times 10^{8} \mathrm{~A} \mathrm{~m}^{-1} \mathrm{~s}^{-1}\right)^{29}$ commonly accepted for whole-body exposure, but all of them are within the less restrictive threshold $\left(5 \times 10^{9} \mathrm{~A} \mathrm{~m}^{-1} \mathrm{~s}^{-1}\right)$ allowed when the region of the body exposed to the AMF is small.

In Fig. 5A three examples of $T(t)$ curves acquired for the most promising sample, S2, (data not shown for sample S1) with different $f H$ combinations are reported, showing a temperature increase of a few Celsius degrees after 5 minutes of exposition to the AFM field. The low temperature increase is due to the low 

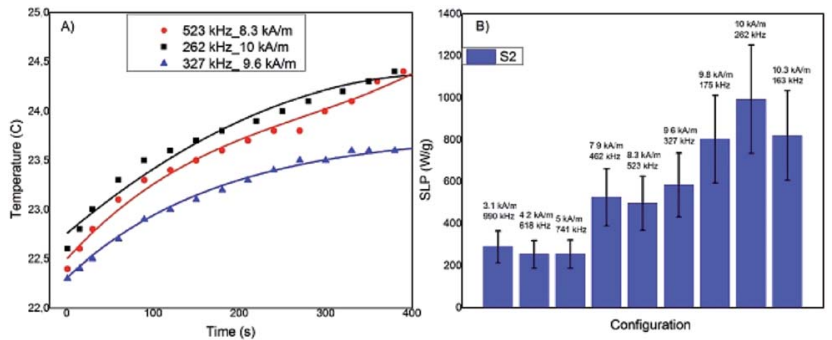

Fig. 5 Hyperthermic efficacy study of sample S2. In (A) the temperature increment for three couples $\left(\mu_{0} H_{0} f\right)$ values are reported, as example. The solid line represent the Box-Lucas fit. In (B) the SLP value for all field-frequency combinations allowed by our apparatus are shown.

MNP concentration used for reducing the interparticles interactions. The SLP values have been evaluated using the BoxLucas function, $T(t)=A\left(1-\mathrm{e}^{B t}\right)$, the most appropriate model when the temperature increment is small, as in this case (examples of the best-fitting curves are shown in Fig. 5A). The SLP results for all the considered $f H$ combinations are reported in Fig. 5B. The SLP values clearly show an increment when the field is increased. The maximum SLP values, about $900 \mathrm{~W} \mathrm{~g}^{-1}$, was obtained for field intensities of the order of $10 \mathrm{kA} \mathrm{m}^{-1}$ in the frequency range $100-300 \mathrm{kHz}$. The high SLP values demonstrate that the new synthetized MNPs are promising hyperthermic agents.

\section{Conclusions}

In this work, we synthesized stable suspensions of MNPs with $d \sim 20 \mathrm{~nm}$ diameter by decomposition of iron pentacarbonyl and further growth with iron oleate. By tuning the reaction conditions, we have been able to prepare two samples having a similar size but a different shape, almost spherical (A/S1) and faceted (A/S2). Further on, transferring the MNPs in water phase by PAMAM coating, at room temperature, preserved the structural properties of the inorganic core. The MNPs have been fully characterized by SQUID magnetometry, displaying a high magnetization, especially for the faceted sample, $\mathrm{S} 2$. In addition, the transverse relaxivities of this sample $-r_{2} \sim 300 \mathrm{mM}^{-1} \mathrm{~s}^{-1}$ at $60 \mathrm{MHz}$ (corresponding to the clinical field 1.5 Tesla) and $r_{2} \sim 250 \mathrm{mM}^{-1} \mathrm{~s}^{-1}$ at $8.5 \mathrm{MHz}$ (corresponding to the field 0.2 Tesla)-are higher than S1 and about 2.5-3 times higher than the corresponding ones of the commercial compound Endorem. Thus, the faceted MNPs underwent calorimetric measurements aimed to verify their possible application in Magnetic Fluid Hyperthermia. The SLP was recorded for several combinations of amplitude and frequency of the applied field and very high values were found at any field intensity and frequency, reaching $900 \mathrm{~W} \mathrm{~g}^{-1}$ at $262 \mathrm{kHz}$ and $10 \mathrm{kA} \mathrm{m}^{-1}$ (within the tolerance limit for $H^{*} f \sim 2.6 \times 10^{9} \mathrm{~A} \mathrm{~m}^{-1} \mathrm{~s}^{-1}$ ), a value indicating these materials among the most promising ones for application in this field. Faceted MNPs being very effective both as MRI negative CAs and hyperthermic mediators, can be envisaged for a future application as theranostic agents, capable of diagnosing cancer, treating it and monitoring the response to treatment. Future investigations will be needed to transfer these results in vivo and, possibly, to properly functionalize the MNPs surface thus addressing specific molecular targets (like e.g. tumour antigenes). Their use as targeting agents will require the achievement of sufficiently long lifetime in the human circulatory system and reduction of doses. The selective delivery to malignant tumor tissues will be pursued through the conjugation with nucleic acids (aptamers), peptides, and natural molecules. On this purpose, the flexibility displayed by the coating chosen for this work seems to offer a quite wide range of possibilities.

\section{Conflicts of interest}

There are no conflicts to declare.

\section{Acknowledgements}

We wish to thank Fondazione Cassa di Risparmio di Firenze for funding this work. The COST European projects RADIOMAG (TD 1402) and EURELAX (CA 15209) are gratefully acknowledged.

\section{Notes and references}

1 M. Franckena, L. J. A. Stalpers, P. C. M. Koper, R. G. J. Wiggenraad, W. J. Hoogenraad, J. D. P. van Dijk, C. C. Wárlám-Rodenhuis, J. J. Jobsen, G. C. van Rhoon and J. van der Zee, Int. J. Radiat. Oncol., Biol., Phys., 2008, 70, 1176-1182.

2 E. L. Jones, J. R. Oleson, L. R. Prosnitz, T. V. Samulski, Z. Vujaskovic, D. Yu, L. L. Sanders and M. W. Dewhirst, J. Clin. Oncol., 2005, 23, 3079-3085.

3 J. van der Zee, D. González, G. C. van Rhoon, J. D. P. van Dijk, W. L. J. van Putten and A. A. M. Hart, Lancet, 2000, 355, 11191125.

4 M. Amichetti, M. Romano, L. Busana, A. Bolner, G. Fellin, G. Pani, L. Tomio and R. Valdagni, Radiother. Oncol., 1997, 45, 155-158.

5 Y. Hua, S. Ma, Z. Fu, Q. Hu, L. Wang and Y. Piao, Int. J. Hyperthermia, 2011, 27, 180-186.

6 N. G. Huilgol, S. Gupta and R. Dixit, Int. J. Hyperthermia, 2010, 26, 21-25.

7 M. E. Sharafinski, R. L. Ferris, S. Ferrone and J. R. Grandis, Head Neck, 2010, 32, 1412-1421.

8 S. Dutz and R. Hergt, Nanotechnology, 2014, 25, 452001.

9 R. Ivkov, S. J. DeNardo, W. Daum, A. R. Foreman, R. C. Goldstein, V. S. Nemkov and G. L. DeNardo, Clin. Cancer Res., 2005, 11, 7093s-7103s.

10 P. Moroz, S. K. Jones and B. N. Gray, Int. J. Hyperthermia, 2002, 18, 267-284.

11 S. Müller, Nanomedicine, 2009, 5, 387-393.

12 J. M. Provenzale and G. A. Silva, Am. J. Neuroradiol., 2009, 30, 1293-1301.

13 K. Maier-Hauff, F. Ulrich, D. Nestler, H. Niehoff, P. Wust, B. Thiesen, H. Orawa, V. Budach and A. Jordan, J. NeuroOncol., 2011, 103, 317-324. 
14 Y. Jin, C. Jia, S.-W. Huang, M. O'Donnell and X. Gao, Nat. Commun., 2010, 1, 41.

15 A. Boni, L. Albertazzi, C. Innocenti, M. Gemmi and A. Bifone, Langmuir, 2013, 29, 10973-10979.

16 A. Boni, D. Ceratti, A. Antonelli, C. Sfara, M. Magnani, E. Manuali, S. Salamida, A. Gozzi and A. Bifone, Contrast Media Mol. Imaging, 2014, 9, 229-236.

17 Y. Yuan, D. Rende, C. L. Altan, S. Bucak, R. Ozisik and D.-A. Borca-Tasciuc, Langmuir, 2012, 28, 13051-13059.

18 A. Boni, G. Bardi, A. Bertero, V. Cappello, M. Emdin, A. Flori, M. Gemmi, C. Innocenti, L. Menichetti, C. Sangregorio, S. Villa and V. Piazza, Nanoscale, 2015, 7, 7307-7317.

19 A. Cervadoro, C. Giverso, R. Pande, S. Sarangi, L. Preziosi, J. Wosik, A. Brazdeikis and P. Decuzzi, PLoS One, 2013, 8, e57332.

20 T. Hyeon, S. S. Lee, J. Park, Y. Chung and H. B. Na, J. Am. Chem. Soc., 2001, 123, 12798-12801.

21 A. Tomalia Donald, S. Uppuluri, R. Swanson Douglas and J. Li, Journal, 2000, 72, 2343.
22 A. H. Morrish, The Physical Principles of Magnetism, John Wiley and Sons, New York, 1965.

23 A.-H. Lu, E. L. Salabas and F. Schüth, Angew. Chem., Int. Ed., 2007, 46, 1222-1244.

24 A. Ouakssim, S. Fastrez, A. Roch, S. Laurent, Y. Gossuin, C. Piérart, L. Vander Elst and R. N. Muller, J. Magn. Magn. Mater., 2004, 272-276, E1711-E1713.

25 A. Roch, R. N. Muller and P. Gillis, J. Chem. Phys., 1999, 110, 5403-5411.

26 M. Basini, T. Orlando, P. Arosio, M. F. Casula, D. Espa, S. Murgia, C. Sangregorio, C. Innocenti and A. Lascialfari, J. Chem. Phys., 2017, 146, 034703.

27 T. Orlando, M. Albino, F. Orsini, C. Innocenti, M. Basini, P. Arosio, C. Sangregorio, M. Corti and A. Lascialfari, J. Appl. Phys., 2016, 119, 134301.

28 Q. L. Vuong, J.-F. Berret, J. Fresnais, Y. Gossuin and O. Sandre, Adv. Healthcare Mater., 2012, 1, 502-512.

29 D. Ortega and Q. A. Pankhurst, in Nanoscience: Volume 1: Nanostructures through Chemistry, The Royal Society of Chemistry, 2013, vol. 1, pp. 60-88. 